\title{
Carcinoma of the External Auditory Canal and Middle Ear: Staging and Management Dilemma
}

\author{
SUNG HUHN KIM, JIN KIM, SA MYUNG CHUNG, WON SANG LEE \\ Department of Otorhinolaryngology, Yonsei University College of Medicine, Seoul, Korea
}

Summary: Objectives: This study aims to figure out more accurate staging and proper therapeutic strategy for carcinoma of external auditory canal and middle ear.

Study Design: Retrospective review of medical records of patients who were diagnosed as squamous cell carcinoma or adenoid cystic carcinoma of external auditory canal and middle ear between 1989 and 2006.

Materials and Methods: 34 patients were enrolled in this study. First, treatment outcomes according to pathologies and stages were investigated. Second, surgical outcomes according to different tumor extent and surgical methods in the same stage were investigated. Third, survival rate according to local invasion to adjacent structures such as parotid gland, facial nerve, temporomandibular joint, infratemporal fossa, dura and intracranium, all of which had been frequently used in other staging systems was investigated. Fourth, the effect of adjuvant radiotherapy in advanced stage (stage III, IV) was investigated by comparing the survival rate between the patients who underwent radiotherapy with the patients who did not undergo radiotherapy.

Results: Patients of stage I and II showed 90\% disease control rates with lateral temporal bone resection (LTBR). However, the survival rates were significantly decreased in stage III and IV. In stage III, LTBR was performed in patients whose tumor was confined to the external auditory canal and middle ear and subtotal temporal bone resection was performed in patients with tumor extending into the mastoid. The difference in disease control rates of the two groups were not significant $(\mathrm{p}>0.05)$. The disease control rates were significantly decreased in the patients with ACC where parotid gland invasion was present $(p=0.007)$ and in the patients with SCC where dural and intracranial invasion was present $(p=0.001)$. There was no significant survival difference between the patients who underwent adjuvant radiotherapy and the patients who did not $(\mathrm{p}>0.05)$.

Conclusions: For the staging system of carcinoma of external auditory canal and middle ear, it can be concluded that staging system needs to be modified. Stage I and II can be put together since the tumors of those stages showed excellent disease control rates. And stage III tumors confined to the external auditory canal and middle ear can be subdivided into two groups, some of which could be included in earlier stage. For the treatment, LTBR seems to be sufficient for stage I, II and limited cases of stage III where tumor is confined to the external auditory canal and middle ear only. In more advanced stages, adjuvant radiotherapy hardly showed any benefit, and therefore initial surgical treatment is more important.

Correspondence: Won Sang Lee, M.D.,

Department of Otorhinolaryngology, Yonsei University College of Medicine

134 Sinchon-dong, Seodaemun-gu, Seoul 120-749, Korea

e-mail: wsleemd@yuhs.ac 
WON SANG LEE : Suggestion of proper staging system and treatment for carcinoma of external auditory canal and middle ear

Key words: carcinoma, external auditory canal, middle ear, staging system

[Accepted Dec. 11, 2007]

\section{INTRODUCTION}

Carcinoma of the external auditory canal and middle ear is a rare disease with a prevalence of $1-6 / 1,000,000 /$ year $^{1)}$. Treatment of the carcinoma was first reported in $1890^{2)}$, and the technical innovation of en bloc resection was accomplished since $1950 \mathrm{~s}^{3)}$. However, treatment is still very difficult and prognosis remains poor, because of the rare incidence, which leads to limited experience, late diagnosis and no coherent staging system of the tumor.

Staging systems for the carcinoma of the external auditory canal and middle ear have been described in many literatures ${ }^{4-8}$, but, unfortunately, there are no universal one. For example, facial nerve invasion is categorized into T4 in modified Pittsburg staging system ${ }^{8}$, which is most commonly used recently, but it is classified as stage $\mathbb{I I}$, not $\mathrm{IV}$, in the Otology group classification ${ }^{6)}$ and is categorized into $\mathrm{T} 2$ in the classification of Clark et $\mathrm{al}^{5)}$. Also, temporomandibular joint invasion is classified as T4, stage II and T3 in modified Pittsburg staging system ${ }^{8)}$, Otology group classification ${ }^{6)}$ and the classification of Clark et $\mathrm{al}^{5}$, respectively. One can find that parotid gland invasion is also classified into different stages in each staging system (Table 1).

A number of studies have suggested different treatment methods of the carcinoma, but conclusion to date is that the best results can be obtained with en bloc resection of the tumor together with radiotherapy ${ }^{9-11)}$. However, there are no universally acceptable staging systems which means the surgical extent can be different depending on which system was used. Adjuvant radiotherapy has been reported to improve the survival of the patients ${ }^{11,12}$, but, in some reports, it was described that radiotherapy did not benefit those with incomplete tumor resection ${ }^{4,13)}$.

In this study, we investigated the treatment outcomes of the tumor according to pathologies, modified Pittsburg staging system and surgical extent. The correlation between treatment outcomes and invasion to adjacent structures of those, which were differently described in many staging systems were also investigated. Finally, we investigated the effectiveness of adjuvant radiotherapy in the tumors of advanced stages and propose some complementary points on staging system and treatment of the external auditory canal and middle ear carcinoma.

\section{PATIENTS AND METHODS}

\section{Patients}

34 patients (15 males, 19 females, mean age 54 years, range 29-77 years) whose histopathology were reported to be squamous cell carcinoma (SCC) or adenoid cystic carcinoma (ACC) were

Table 1 Current status of staging system

\begin{tabular}{lccc}
\hline Prognostic factor & $\begin{array}{c}\text { Modified Pittsburg } \\
(2000)\end{array}$ & $\begin{array}{c}\text { The Otology Group } \\
(1999)\end{array}$ & Clark/Stell (1991) \\
\hline Facial nerve & T4 & III & T2 \\
TM joint & T4 & II & T3 \\
Parotid & - & II & T3 \\
\hline
\end{tabular}


J Jpn Soc Head Neck Surg 17 (3) : 193 201, 2007

Table 2 Characteristics of patients with squamous cell carcinoma of the external auditory canal and middle ear

\begin{tabular}{|c|c|c|c|c|c|c|}
\hline Patient & Sex/Age & $\begin{array}{l}\text { Preoperative } \\
\text { stage }\end{array}$ & Surgery & RT & Status & (Mo) \\
\hline 1 & $\mathrm{M} / 61$ & I & LCE & None & NED & (138) \\
\hline 2 & $\mathrm{M} / 68$ & I & LTBR & Adjuvant & NED & (28) \\
\hline 3 & $\mathrm{M} / 37$ & I & LTBR & None & NED & $(45)$ \\
\hline 4 & $\mathrm{M} / 67$ & I & LTBR & None & NED & (36) \\
\hline 5 & $\mathrm{~F} / 67$ & II & LTBR & None & NED & (28) \\
\hline 6 & $\mathrm{M} / 67$ & II & LTBR & None & NED & $(62)$ \\
\hline 7 & $\mathrm{~F} / 60$ & II & LTBR & None & AWD & $(24)$ \\
\hline 8 & $\mathrm{~F} / 67$ & III & LTBR & None & NED & (28) \\
\hline 9 & $\mathrm{~F} / 64$ & III & STBR & Adjuvant & NED & (111) \\
\hline 10 & $\mathrm{M} / 56$ & III & STBR & None & DOD & (3) \\
\hline 11 & $\mathrm{~F} / 49$ & III & STBR & Adjuvant & DOD & $(32)$ \\
\hline 12 & $\mathrm{~F} / 43$ & IV & STBR & Adjuvant & NED & (12) \\
\hline 13 & $\mathrm{M} / 57$ & IV & STBR & None & NED & (19) \\
\hline 14 & $\mathrm{M} / 47$ & IV & STBR & Adjuvant & NED & (18) \\
\hline 15 & $\mathrm{M} / 62$ & IV & STBR & Adjuvant & AWD & (24) \\
\hline 16 & $\mathrm{~F} / 56$ & IV & STBR & None & AWD & $(12)$ \\
\hline 17 & $\mathrm{~F} / 77$ & IV & STBR & Adjuvant & DOD & $(24)$ \\
\hline 18 & $\mathrm{M} / 61$ & IV & STBR & Adjuvant & DOD & (7) \\
\hline 19 & $\mathrm{M} / 48$ & IV & STBR & Adjuvant & DOD & $(29)$ \\
\hline 20 & $\mathrm{M} / 56$ & IV & STBR & Adjuvant & DOD & (34) \\
\hline 21 & $\mathrm{M} / 35$ & IV & TTBR & None & DOD & (6) \\
\hline
\end{tabular}

${ }^{*} \mathrm{LCE}=$ Local canal excision, $\mathrm{LTBR}=$ Lateral temporal bone resection,

$\mathrm{STBR}=$ Subtotal temporal bone resection, $\mathrm{TTBR}=$ Total temporal bone resection,

${ }^{*} \mathrm{NED}=$ No evidence of disease, $\mathrm{AWD}=$ Alive with disease, $\mathrm{DOD}=$ Dead of disease.

enrolled in this study. They underwent various types of temporal bone resections between March 1989 and January 2006 at the Severance hospital, Yonsei University. Mean follow-up period for the patients was 37 months.

Histopathological examinations confirmed 21 cases of SCC and 13 cases of ACC. Tumors were staged preoperatively according to the modified Pittsburg systems ${ }^{8)}$ by reviewing the medical records and imaging studies of the patients. There were four stage I patients, three stage II patients, four stage III patients and ten stage IV patients in SCC group (Table 2). In the ACC group, there were one stage 1 patient, two stage II patients, five stage III patients and five stage IV patients (Table 3 ).

Treatment

Surgical treatment was performed in all patients as an initial treatment. The surgical procedures were defined and performed as follows: Local canal excision (LCE) was defined as removal of the skin and all or part of the cartilaginous external auditory canal and may have included removal of a portion of the bony canal, which was performed in one case of stage I SCC patient. Lateral temporal bone resection (LTBR) was defined as the total removal of bony and cartilageous part of external auditory canal including ear drum, malleus, and incus after complete mastoidectomy (Fig. 1A), which was performed in stage I, II and limited cases of stage III patients where the tumor was confined only to the external auditory canal and middle ear. Subtotal temporal bone resection (STBR) 
Table 3 Characteristics of patients with adenoid cystic carcinoma of the external auditory canal and middle ear

\begin{tabular}{|c|c|c|c|c|c|c|}
\hline Patient & Sex/Age & $\begin{array}{l}\text { Preoperative } \\
\text { stage }\end{array}$ & Surgery & RT & Status & $(\mathrm{Mo})$ \\
\hline 1 & $\mathrm{~F} / 47$ & I & LTBR & None & NED & (48) \\
\hline 2 & $\mathrm{M} / 52$ & II & LTBR & Adjuvant & NED & (75) \\
\hline 3 & $\mathrm{~F} / 71$ & II & LTBR & None & NED & (124) \\
\hline 4 & $\mathrm{~F} / 49$ & III & LTBR & Adjuvant & NED & (49) \\
\hline 5 & $\mathrm{~F} / 50$ & III & LTBR & None & AWD & $(70)$ \\
\hline 6 & $\mathrm{M} / 45$ & III & STBR & None & NED & (18) \\
\hline 7 & $\mathrm{M} / 52$ & III & STBR & Adjuvant & DOD & (65) \\
\hline 8 & $\mathrm{~F} / 60$ & III & STBR & None & DOD & (98) \\
\hline 9 & $\mathrm{~F} / 60$ & IV & STBR & Adjuvant & AWD & $(25)$ \\
\hline 10 & $\mathrm{~F} / 50$ & IV & STBR & None & AWD & (99) \\
\hline 11 & $\mathrm{~F} / 67$ & IV & STBR & Adjuvant & AWD & $(50)$ \\
\hline 12 & $\mathrm{~F} / 49$ & IV & STBR & Adjuvant & DOD & (25) \\
\hline 13 & $\mathrm{~F} / 53$ & IV & STBR & Adjuvant & DOD & (21) \\
\hline
\end{tabular}

${ }^{*} \mathrm{LCE}=$ Local canal excision, $\mathrm{LTBR}=$ Lateral temporal bone resection,

STBR $=$ Subtotal, temporal bone resection, $\mathrm{TTBR}=$ Total temporal bone resection,

${ }^{*} \mathrm{NED}=$ No evidence of disease, $\mathrm{AWD}=$ Alive with disease, $\mathrm{DOD}=$ Dead of disease.
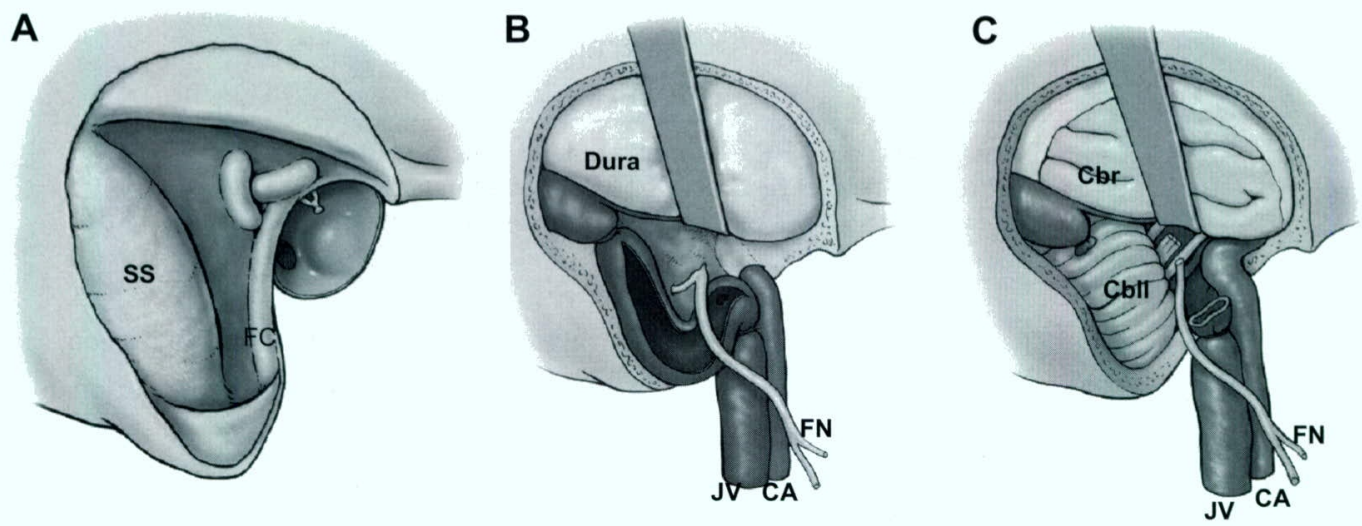

SS: Sigmoid sinus, FC: Facial canal, JV: Jugular vein, CA: Carotid artery, FN: Facial nerve, Cbr: Cerebrum, Cbll: Cerebellum

Fig. 1 Various types of temporal bone resection which was performed in this series.

(A) Lateral temporal bone resection was defined as the total removal of bony and cartilageous part of external auditory canal including ear drum, malleus, and incus after complete mastoidectomy. (B) Subtotal temporal bone resection was defined as removal of nearly all of the temporal bone including inner ear except the petrous apex. (C) Total temporal bone resection was defined as removal of the total temporal bone including petrous apex, dura, seventh and eighth nerves in one piece, while saving carotid artery.

was defined as removal of nearly all of the temporal bone including inner ear except the petrous apex (Fig. 1B), which was performed in most stage III and IV patients. Total temporal bone resection (TTBR) defined as removal of the total temporal bone including petrous apex, dura, sigmoid sinus, seventh and eighth cranial nerves in one piece, while saving carotid artery (Fig. 1C), which was performed only in one stage IV patient. 


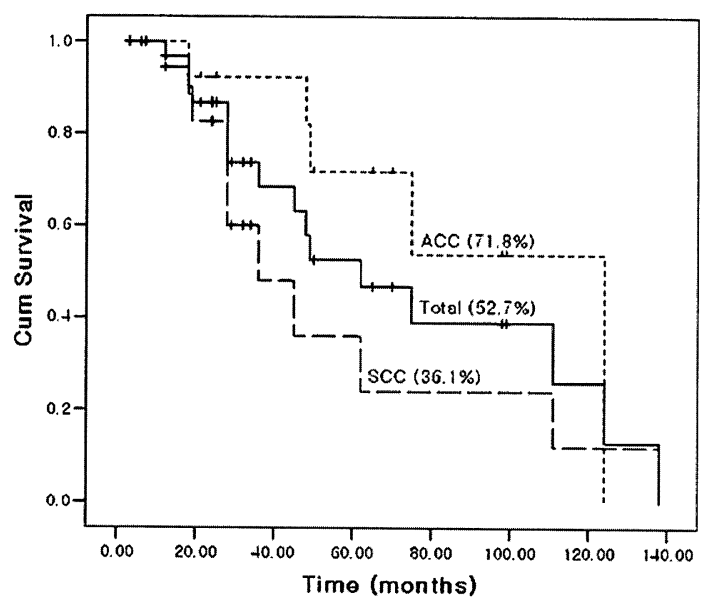

Fig. 2 The estimated disease-free 5-year survival rates for carcinoma of external auditory canal and middle ear.

Total parotidectomy was performed when the tumor definitely involved the parotid gland in preoperative evaluation. Otherwise, superficial parotidectomy was performed.

Adjuvant radiotherapy was carried out in patients who had positive surgical margin in final pathology report and in advanced stage (III, IV) patients. Eighteen patients underwent radiotherapy, but nine patients in advanced stages did not. (Table 2 and 3 ).

Methods

First, treatment outcomes according to pathologies and stages were investigated. Second, surgical outcomes according to different tumor extent and surgical methods in the same stage were investigated. We performed LCE and LTBR in stage I patients, and STBR and TTBR in stage IV patients, but LCE and TTBR were performed only in one case of each stages. Therefore, the analysis was focused on the surgical outcomes in stage III where LTBR and STBR were performed according to the tumor extent. Third, survival rate according to adjacent structure invasion such as parotid gland, facial nerve, temporomandibular joint, infratemporal fossa, dura and intracranium, all of which were frequently used in other staging systems was investigated. Fourth, the effect of adjuvant radiotherapy in advanced stage (stage III, IV) was investigated by comparing the survival between the patients who underwent radiotherapy $(\mathrm{n}=15)$ and the patients who did not undergo radiotherapy $(n=9)$. Kaplan-Meier method was used to get the survival rate curve. Log-rank test was used for the univariate analysis of significance of each factor and Cox proportional hazard model was used to assess the simultaneous significance of each factor for survival. Statistical analysis was performed with SPSS 13.0 for windows (SPSS Inc, Chicago, IL) and a value of $p<0.05$ was considered to be significant statistically.

\section{RESULTS}

Treatment outcomes according to pathologies and stages

The estimated disease-free five-year survival rates were $52.7 \%$ overall, and $36.1 \%$ for SCC and $71.8 \%$ for ACC respectively (Fig. 2). Disease-free 5-year survival rates between SCC and ACC patients were not significantly different $(p>0.05)$. The more stage advanced, the further survival 


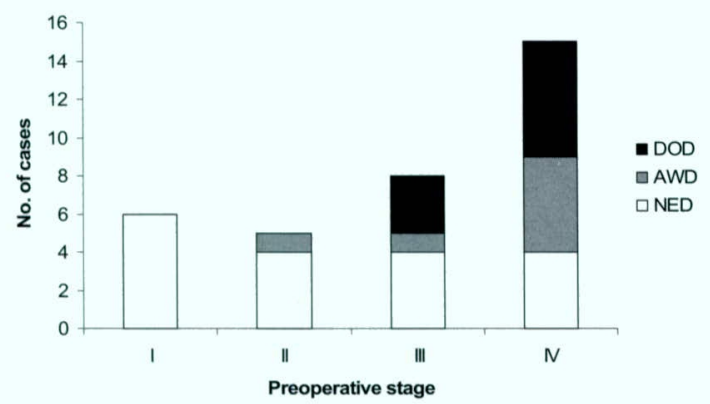

NED: No evidence of disease, AWD: Alive with disease, DOD: Dead of disease.

Fig. 3 Survival status according to stage. Patients of stage I showed $100 \%$ survival rates and, in stage II, total of four out of five are disease-free status. The survival rates were significantly decreased in stage III and IV and disease control rate of stage III and IV were $50 \%(4 / 8)$ and $29 \%(4 / 14)$, respectively.

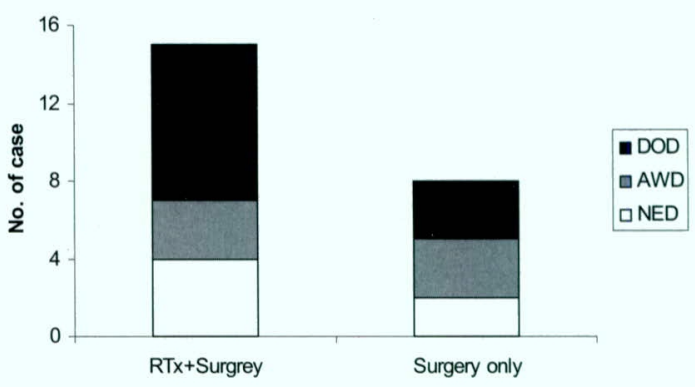

NED: No evidence of disease, AWD: Alive with disease, DOD: Dead of disease.

Fig. 4 Survival status of patients in stage III and IV according to adjuvant radiotherapy.

rate decreased in each of pathology. Patients of stage I showed $100 \%$ survival rates and, in stage II, total of four out of five were disease-free status. However, the survival rates were significantly decreased in stage III and IV. Disease control rate of stage III and IV were 50\% (4/9) and 29\% (3/15), respectively (Fig. 3).

Treatment outcomes in carcinoma of stage III

Stage III carcinoma was divided into two groups according to the extent of invasion. In one group, the tumor was confined to the external auditory canal and middle ear and all patients received LTBR. There were three patients, one was SCC patient and two were ACC patients. Two patients (SCC, ACC) are alive without evidence of disease and one patient (ACC) is alive with disease (Table $2 \& 3$ ). Their mean follow-up periods are forty nine months (range: 28-70). In the other group, the tumor extended into the middle ear, mastoid air cells and soft tissues which consequently led to STBR. There were six patients, three were SCC patients and three were ACC patients. Four patients died of disease and two patients are alive without evidence of disease (Table $2 \& 3$ ). The disease control rate was significantly different between the two groups $(\mathrm{p}<0.05)$.

Treatment outcomes according to adjacent tissue invasion

We have analyzed the survival rate depending on invasion of surrounding structures of EAC such as parotid gland, facial nerve, TMJ, ITF and dura. The result in cases of parotid gland invasion, multivariate analysis showed no significant difference in the survival rate in SCC patients, when significant difference was observed in ACC patients $(\mathrm{p}=0.007)$. TMJ invasion in SCC patients, ITF invasion in ACC patients, and Facial nerve invasion in both patient groups presented with significant decrease in survival rate with univariate analysis $(\mathrm{p}<0.05)$, but they were not statistically significant when multivariate analysis was used. In cases of dura invasion, there was significant decrease in the survival rate in SCC patients with multivariate analysis $(\mathrm{p}=0.001$ ) (Table 4).

Therapeutic effect of adjuvant radiotherapy on patients with the advanced stage-tumors

In fifteen patients who underwent adjuvant radiotherapy, disease control failed in eight patients. 
Table 4 3-year survival rate according to adjacent tissue invasion. Parotid gland invasion and dura involve involvement showed significantly decreased survival rates in ACC and SCC patients, respectively, in multivariate analysis. In each cell remark number of alive patients among total patients.

\begin{tabular}{lcccccc}
\hline & \multicolumn{2}{c}{ SCC } & \multirow{2}{*}{$\begin{array}{c}\text { P-value } \\
\end{array}$} & Invasion (+) & Invasion $(-)$ & \multicolumn{2}{c}{ ACC } & \multirow{2}{*}{$\begin{array}{c}\text { P-value } \\
(\text { MV }\end{array}$} & & Invasion $(+)$ & Invasion $(-)$ & \\
\hline Parotid gland* & $3 / 3$ & $11 / 18$ & $\mathrm{p}>0.05$ & $4 / 6$ & $5 / 7$ & $\mathrm{P}=0.007$ \\
TMJ & $4 / 9$ & $11 / 12$ & $\mathrm{p}>0.05$ & $3 / 4$ & $7 / 9$ & $\mathrm{p}>0.05$ \\
ITF & $0 / 1$ & $14 / 20$ & $\mathrm{p}>0.05$ & $1 / 3$ & $8 / 10$ & $\mathrm{p}>0.05$ \\
Facial nerve & $2 / 4$ & $12 / 17$ & $\mathrm{p}>0.05$ & $0 / 2$ & $9 / 11$ & $\mathrm{p}>0.05$ \\
Dura* & $3 / 4$ & $11 / 17$ & $\mathrm{P}=0.001$ & $2 / 4$ & $7 / 9$ & $\mathrm{p}>0.05$ \\
\hline
\end{tabular}

SCC: Squamous cell carcinoma, ACC: Adenoid cystic carcinoma, MV: Multivariate analysis,

TMJ: Temporomandibular joint, ITF: Infratemporal fossa.

In SCC,

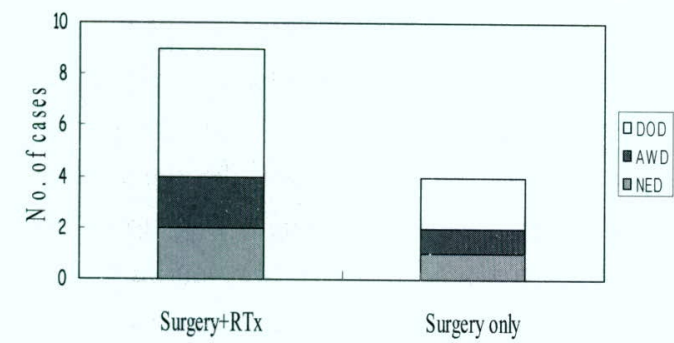

In ACC,

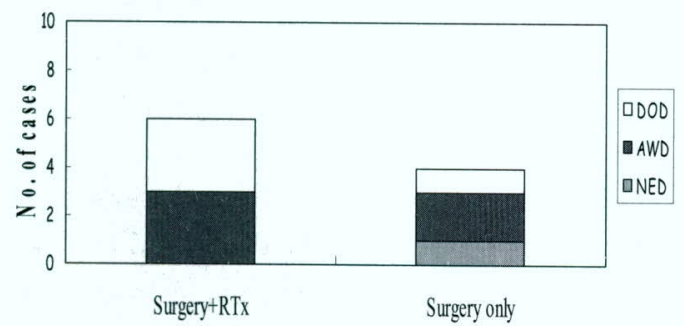

SCC: Squamous cell carcinoma, ACC: Adenoid cystic carcinoma, NED: No evidence of disease,

AWD: Alive with disease, DOD: Dead of disease.

Fig. 5 Survival of Adjuvant radiotherapy vs. Surgery alone

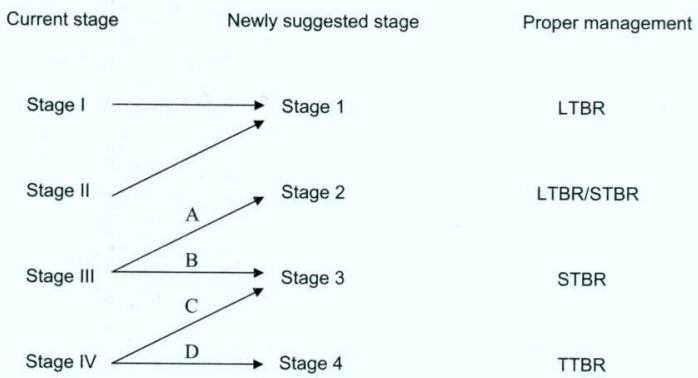

A: invasion of middle ear only

$B$ : invasion of middle ear and mastoid

$\mathrm{C}$ : invasion of facial nerve, temporomandibular joint and parotid gland

$\mathrm{D}$ : invasion of intracranium, labyrinth and distant metastasis

LTBR $=$ Lateral temporal bone resection,

STBR $=$ Subtotal temporal bone resection,

TTBR $=$ Total temporal bone resection .

Fig. 6 Proposal of new staging system

Only four patients are alive without evidence of disease, whereas three patients are alive with disease and eight patients died of disease. Disease control also failed in six out of eight patients who did not undergo adjuvant radiotherapy; three patients are alive with disease and the other three patients died of disease (Table 2, Fig. 5). There was no significant difference in survival rate between the two groups $(\mathrm{p}>0.05)$. We could not find any difference in survival rate between the two groups when we compared them in the aspect of two different pathologies. (Fig. 6) The mean follow-up periods of patients alive were 41 months (range 12-111 months) 


\section{DISCUSSION}

1. Stage I, II carcinoma: Could it be categorized into same stage?

In the recent reports, 5 -year survival rates of external auditory canal and middle ear carcinoma has been described as $45-61 \%{ }^{4,11,14)}$. Treatment outcomes for the tumors of early stages (stage I, II) have been reported to be excellent, however those for advanced stages (stage III, IV) have been reported to be extremely poor, which have not changed significantly since 1980s. This fact is also noted in our series. Disease control rate was excellent in stage I and II, but it was significantly decreased in stage III, IV regardless of the reported pathology. In stage I, II, all patients are alive without evidence of disease except one who is alive with disease. Although, the number of patients of stage I and II was small $(n=10)$, we propose carefully that stage I and II could be categorized into the same stage because the treatment methods for them were same (LTBR and adjuvant radiotherapy in cases of positive surgical margin) and disease-free survival rate was nearly $100 \%$.

2. Stage III carcinoma: Is subclassification necessary?

Recent studies advocate en bloc surgery with adjuvant radiotherapy as the treatment of choice for external auditory canal and middle ear carcinoma ${ }^{9-11)}$. Usually, they recommend LTBR for lesions confined to the external auditory canal (stage I, II ) and STBR/TTBR for the lesions which extend beyond the external auditory canal (stage II, IV). The prognosis falls so dramatically with middle ear involvement that extensive surgery such as STBR/TTBR has been preferred ${ }^{1}$. However, in the study of Prasad and Janecka ${ }^{15)}$, the 5-year survival rates were not different statistically between the patients who underwent LTBR and the patients who underwent STBR with the tumor extending into the middle ear. In this study, we performed LTBR in patients where the tumor confined to the external auditory canal and middle ear without extension to mastoid air cells. The disease control rates were better than those patients whose tumor extended to mastoid air cells and STBR was performed. Although we could not conclude that the tumors extending only to middle ear can be subclassified from stage III due to small number of cases, we can insist that LTBR can be a good treatment of choice in the tumors of stage III which is confined to external auditory canal and middle ear.

3. Tumor characteristics according to pathology: Should it be considered in staging and treatment? Parotid gland invasion and dura/intracranial involvement showed significantly decreased survival rates in ACC and SCC patients, respectively, in multivariate analysis, and parotid gland invasion of ACC and dural invasion of SCC which are classified into different stages in each different staging system can be categorized to advanced stage. In univariate analysis (Log-rank test), facial nerve invasion, temporomandibular joint invasion and dura/intracranial invasion decreased survival rates in SCC patients, whereas facial nerve invasion, infratemporal fossa invasion and parotid invasion decreased survival rates in ACC patients. Although we did not show the data in this report, most distant metastasis occurred in advanced stage ACC $(n=4$ [80\%]). Authors have reported previously that direct parotid invasion occurred only in advanced staged SCC, but it did occur in early stage ACC, and parotid node metastasis occurred only in the patients of advanced staged SCC ${ }^{16)}$. As you can see above, characteristics of the tumor seems to be different regarding to pathology. SCC showed tendency to widely destruct surrounding structures and spread more easily through lymphatics (though this is the rare case in carcinoma of external auditory canal and middle ear), whereas ACC more easily spread with neural structures and to the distant organs. Surgeons have to consider the different charateristics of the tumor according to their pathology in planning the treatment. 
4. Adjuvant radiotherapy in advanced staged carcinoma: Is it beneficial?

Nowadays adjuvant radiotherapy is considered as an essential treatment strategy. A number of studies demonstrated it to be beneficial in managing external auditory canal and middle ear carcino$\mathrm{ma}^{9-12)}$. However, there were reports questioning the effect of postoperative radiotherapy in cases of incomplete surgical resection ${ }^{4,13)}$. In this study, adjuvant radiotherapy in advanced staged carcinoma did not show any benefit. We thought that carcinoma of the external auditory canal and middle ear behave so aggressively that it already spreads extensively and widely under the bone in advanced stages. Therefore, radiotherapy might provide little benefit in treating advanced staged carcinoma. This result emphasizes the importance of the initial surgical treatment and one must be prudent in planning the surgical extent.

\section{CONCLUSION}

For the staging system of carcinoma of external auditory canal and middle ear, it can be concluded that staging system needs to be modified. Stage I and II can be put together since the tumors of those stages showed excellent disease control rates. And stage III tumors confined to the external auditory canal and middle ear can be subdivided into several groups, some of which could be included in earlier stage. For the treatment, LTBR seems to be sufficient for stage I, II and limited cases of stage III where tumor is confined to the external auditory canal and middle ear only. In more advanced stages, adjuvant radiotherapy hardly showed any benefits, and therefore initial surgical treatment is more important.

\section{REFERENCES}

1) Barrs DM: Temporal bone carcinoma. Otolaryngol Clin North Am $34: 1197-1218, x, 2001$.

2) Kuhel WI, Hume CR, Selesnick SH: Cancer of the external auditory canal and temporal bone. Otolaryngol Clin North Am 29:827-852, 1996.

3) Parsons H, Lewis JS: Subtotal resection of the temporal bone for cancer of the ear. Cancer $7: 995-1001,1954$.

4) Goodwin WJ, Jesse RH: Malignant neoplasms of the external auditory canal and temporal bone. Arch Otolaryngol $106: 675-679,1980$.

5) Clark LJ, Narula AA, Morgan DA, et al: Squamous carcinoma of the temporal bone: a revised staging. J Laryngol Otol $105: 346-348,1991$.

6) Manolidis S, Pappas D, Jr., Von Doersten P, et al: Temporal bone and lateral skull base malignancy: experience and results with 81 patients. Am J Otol 19 : S1-15, 1998.

7) Arriaga M, Curtin $\mathrm{H}$, Takahashi $\mathrm{H}$, et al: Staging proposal for external auditory meatus carcinoma based on preoperative clinical examination and computed tomography findings. Ann Otol Rhinol Laryngol $99: 714-721,1990$.

8) Moody SA, Hirsch BE, Myers EN: Squamous cell carcinoma of the external auditory canal: an evaluation of a staging system. Am J Otol $21: 582-588,2000$.

9) Conley J, Schuller DE: Malignancies of the ear. Laryngoscope $86: 1147-1163,1976$.

10) Testa JR, Fukuda Y, Kowalski LP: Prognostic factors in carcinoma of the external auditory canal. Arch Otolaryngol Head Neck Surg 123: 720-724, 1997.

11) Pfreundner L, Schwager K, Willner J, et al: Carcinoma of the external auditory canal and middle ear. Int J Radiat Oncol Biol Phys $44: 777-788,1999$.

12) Zhang B, Tu G, Xu G, et al: Squamous cell carcinoma of temporal bone: reported on 33 patients. Head Neck $21: 461-466,1999$.

13) Nyrop M, Grontved A: Cancer of the external auditory canal. Arch Otolaryngol Head Neck Surg $128: 834-$ 837, 2002.

14) Moffat DA, Grey P, Ballagh RH, et al: Extended temporal bone resection for squamous cell carcinoma. Otolaryngol Head Neck Surg 116:617-623, 1997.

15) Prasad S, Janecka IP: Efficacy of surgical treatments for squamous cell carcinoma of the temporal bone: a literature review. Otolaryngol Head Neck Surg $110: 270-280,1994$.

16) Choi JY, Choi EC, Lee HK, et al: Mode of parotid involvement in external auditory canal carcinoma. J Laryngol Otol $117:$ 951-954, 2003. 